\title{
Current-induced domain wall motion in nanoscale ferromagnetic elements
}

\author{
G Malinowski ${ }^{1}$, O Boulle ${ }^{2}$ and M Kläui ${ }^{3,4}$ \\ ${ }^{1}$ Laboratoire de Physique des Solides, CNRS, Université Paris-sud 11, 91405 Orsay Cedex, France \\ ${ }^{2}$ SPINTEC, CEA/CNRS/UJF/GINP, INAC, 38054 Grenoble Cedex 9, France \\ ${ }^{3}$ SwissFEL, Paul Scherrer Institut, 5232 Villigen PSI, Switzerland; Laboratory of Nanomagnetism and \\ Spin Dynamics, Ecole Polytechnique Fédérale de Lausanne (EPFL), 1015 Lausanne, Switzerland \\ E-mail: Klaeui@uni-mainz.de
}

\begin{abstract}
We review the details of domain wall (DW) propagation due to spin-polarized currents that could potentially be used in magnetic data storage devices based on domains and DWs. We discuss briefly the basics of the underlying spin torque effect and show how the two torques arising from the interaction between the spin-polarized charge carriers and the magnetization lead to complex dynamics of a spin texture such as a DW. By direct imaging we show how confined DWs in nanowires can be displaced using currents in in-plane soft-magnetic materials, and that when using short pulses, fast velocities can be attained. For high-anisotropy out-of-plane magnetized wires with narrow DWs we present approaches to deducing the torque terms and show that in these materials potentially more efficient domain wall motion could be achieved.
\end{abstract}

(Some figures in this article are in colour only in the electronic version)

\section{Introduction}

Magnetic nanostructures have in the past been at the heart of a multitude of devices ranging from sensing applications to data storage. Probably the best known storage device is the magnetic disk drive [1], which was pioneered in the 1950s by IBM with the RAMAC and since then the storage density has seen a gigantic exponential increase. While hard drives continue to excel in the high capacity market, they entail nonetheless disadvantages, which have led to other memory concepts replacing them for low capacity storage applications. One of the key problems is the mechanical motion of the media, which poses reliability questions and can lead to catastrophic failure in the case of mechanical shock. Another successful magnetic memory is magnetic tape, which has a huge capacity but obvious limitations when it comes to random access times [2]. Again tape drives physically move the media (tape), which leads to wear. Another disadvantage that physical motion brings with it is the power consumption, which for instance in laptops leads to a significant part of the power being used by the hard drive.

${ }^{4}$ Current address: Institut für Physik, Universität Mainz, Staudinger Weg 7 , 55128 Mainz, Germany.

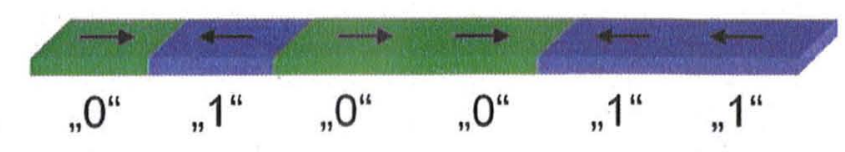

Figure 1. Schematic of a magnetic wire with domains. The colour and the arrows indicate the magnetization direction. The domains with the magnetization pointing to the right correspond here to a logical 0 and magnetization pointing to the left corresponds to a 1 . So in the schematic part of the wire the domains encode the digital information 010011 .

For many of the growing memory markets in particular in mobile applications (laptops, PDAs, etc), low power combined with a solid state technology is required. Different approaches have been suggested based on a range of technologies. For this review we will focus on magnetic materials and thus for magnetic data storage, a paradigm shift away from hard drives or tape is required: one exciting approach that has been recently proposed is based on magnetic nanowires with magnetic domains acting as the bits (figure 1). The interfaces between two domains where the magnetization is pointing in opposite directions is called a magnetic domain wall (DW), in which the spins turn by $180^{\circ}$ and the nanoscale spin structure depends on the material and geometry. At first sight, the 
design looks similar to tape, but rather than shifting the media with the data as in the case of tape, here the data are shifted within the media which stays physically fixed. This leads to much faster possible access times and in particular eliminates all the mechanical motion. On the other hand, the device only comprises one write or read element for potentially tens to hundreds of bits, which can make it cost effective. Furthermore this simplifies the integration with the necessary semiconductor electronics, which can be more compact and can thus lead to higher storage densities, compared with for instance magnetic random access memory [3]. Prominent suggestions for concrete realizations of such a device are the racetrack device envisaged by Parkin [4, 5] and the shift register proposed by Cowburn et al [6].

To make such a device useful for memory storage, three key tasks have to be performed: the data need to be written, read and selected (meaning that the bit to be read or written needs to be moved to the read or write element). The reading and writing approach is similar to the one used in hard drives [7]. However, it is the domain selection by motion of the domains and DWs that is novel and requires alternative approaches. So the dynamic behaviour of geometrically confined DWs and their motion has become a topic of growing interest in recent years due to the intricate magnetic properties present in geometrically confined ferromagnetic structures [8]. The obvious approach to manipulate magnetization and DWs would be to use conventional magnetic fields. Two problems though arise when using magnetic fields as two adjacent DWs (say a head-to-head and a tail-to-tail wall) will move in opposite directions when a field is applied, thus annihilating one another rather than moving the domain in between in the desired direction. Secondly field-induced motion suffers from a serious scaling drawback as the field necessary to move a wall has to stay constant (for thermal stability issues) which means that with decreasing lateral dimensions the striplines that generate the field need to carry an ever increasing current density that will eventually lead to a structural breakdown. An alternative approach is current-induced DW motion due to the spin-transfer torque effect, which we discuss here. A constant current density is needed to move DWs, which means that with decreasing design rule, the power consumption goes down as well paving the way for energy saving devices.

\section{Current-induced domain wall motion basics (CIDM)}

The theory of current-induced DW motion has been treated by a number of excellent theoretical groups. An extensive review can be found in [9] and in this cluster, a paper by Shibata et al reviews the theory as well. Here we only give some details that are needed to understand the experimental observations and interpretations presented later on.

The idea that the transfer of spin from conduction electrons moving across a spin texture of a DW can be used to manipulate the DW was first introduced by Berger at the end of the 1970s [10]. When a current, which is naturally polarized in a metallic ferromagnet, crosses the $\mathrm{DW}$, the exchange interaction aligns the conduction electron spin polarization direction along the direction of the local magnetization. As the exchange interaction conserves the total spin, this angular momentum has to be transferred to the local magnetization, which is equivalent to a torque acting on the magnetization resulting in a DW displacement in the direction of the electron flow. As this effect is independent of the film thickness, it dominates for thin films over the hydrodynamic drag effect that originates from the Lorentz force [11].

The corresponding adiabatic spin torque $\vec{\tau}_{\mathrm{ST}}$ can be converted into a time derivative of the unit magnetization:

$$
\left(\frac{\partial \vec{m}}{\partial t}\right)_{\mathrm{ST}}=-u \frac{\partial \vec{m}}{\partial x}
$$

with $u=J P g \mu_{B} / 2 e M_{\mathrm{s}}$, where $J$ is the current density, $p$ is the current polarization, $g$ is the Lande factor, $\mu_{\mathrm{B}}$ is the Bohr Magneton, $e$ is the electron charge and $M_{\mathrm{s}}$ is the saturation magnetization. $u$ is generally called the spin drift velocity and is actually the maximum velocity that the DW can reach when the conduction electron spin moments are fully converted into DW displacement.

Since theories developed to describe spin-transfer torque in the adiabatic limit were not able to reproduce experimental results observed on nanowires with in-plane magnetization, it was suggested that the effect of spin transfer was more complicated and that nonadiabatic contributions are present. The corresponding nonadiabatic torque was first introduced by Zhang and Li [12] and Thiaville et al [13] and is characterized by a dimensionless parameter $\beta$

$$
\frac{\partial \vec{m}}{\partial t}=\beta \vec{m} \times[(\vec{u} \cdot \nabla) \vec{m}] .
$$

This torque is perpendicular to the adiabatic torque and although its amplitude is expected to be small in $3 \mathrm{~d}$ metal with $\beta_{s r} \approx 0.01$, it significantly alters the dynamics of the DW and in particular can determine the critical current density and the terminal velocity of the DW. Two different contributions have been identified for this torque. The first one is due to the spin relaxation in the DW, $\left(\beta_{s r}\right)[9,12-18]$. Such spin relaxation can occur through spin-flip scattering events with impurities, phonon, etc so that in these processes spin is not conserved due to the spin-orbit interaction. Several authors have carried out calculations to extract the value of $\beta_{\mathrm{sr}}[12,16,17,19-21]$. A second contribution to $\beta$ is a pure nonadiabatic contribution occurring when the spatial gradient of the magnetization is very large for the current spin polarization to follow the local magnetization direction (described by the nonadiabaticity parameter due to nonadiabatic transport $\beta_{\text {na }}$ ) $[18,22-25]$. For more information, see the review paper by Shibata $\mathrm{et} \mathrm{al}$ in this cluster.

To understand the experimental finding, comparison with micromagnetic numerical simulations using the LandauLifshitz-Gilbert equation including the effects of spin currents $[26,27]$ can be used. This equation can be simplified to a set of two coupled differential equations known as the one-dimensional analytical model assuming a constant DW profile and taking as parameters the DW position $q$ and the 
magnetization angle $\psi[13,14,26-29]$ :

$$
\begin{gathered}
\dot{\psi}+\frac{\alpha \dot{q}}{\Delta}=\gamma \mu_{0} H+\frac{\beta u}{\Delta}-\frac{\gamma}{2 M_{\mathrm{s}}} \frac{\partial V_{\text {pin }}}{\partial q} \\
\frac{\dot{q}}{\Delta}-\alpha \dot{\psi}=\frac{\gamma \mu_{0} H_{k}}{2} \sin 2 \psi+\frac{u}{\Delta}
\end{gathered}
$$

where $H_{k}$ is the restoring field for the DW transverse orientation, $H$ is the external magnetic field applied along the easy axis, and $\Delta(\psi)=\left(A /\left(K_{0}+K \sin ^{2} \psi\right)\right)^{1 / 2}$ is the DW width with $K_{0}$ being the uniaxial longitudinal anisotropy and $K=\mu_{0} M_{\mathrm{s}} H_{k} / 2$ is the transverse anisotropy mainly due to magnetostatic effects [30]. $V_{\text {pin }}$ is the pinning potential that may depend on $q$. One sees that the nonadiabatic torque enters equation (3) with an analytical form similar to an external field $H_{I}$ with $\mu_{0} H_{I}=\beta u / \Delta \gamma$.

This simple 1D model can be used to deduce the critical current and the DW velocity when the motion is driven by current in the absence of pinning (perfect wire with no roughness) and from comparison with the experiment key parameters can be deduced. For details of the model, see the review by Shibata et al in this cluster.

For $\beta=0$, there is a threshold current value below which there is no steady DW motion [12] and above a critical current density, the DW starts moving. This threshold value corresponds to the onset of periodic DW transformations [31-33].

As mentioned above, the nonadiabatic torque acts as a magnetic field that can sustain steady-state DW motion resulting in the disappearance of the intrinsic threshold current even for very small values of $\beta$, the real threshold current for DW motion being only determined by extrinsic pinning. The DW velocity in the steady state regime increases linearly as $v_{\text {final }}=\beta u / \alpha$ up to a critical velocity $[12,15,32]$.

Recently, the inclusion of thermal effects on the currentinduced DW dynamics started to be studied by different authors [34-45]. The effect of thermal activation on the magnetization dynamics is generally included by adding to the Landau-Lifschitz-Gilbert equation a stochastic Gaussian distributed magnetic field [46] with zero mean value and correlation. The previous approaches using the modified LLG equation lack the correct description of temperature effects because of the assumption of a constant magnetization length. Schieback et al [43] employed the so-called Landau-Lifschitz-Bloch (LLB) equation which implements a macrospin model with longitudinal relaxation processes to investigate realistic temperature effects. They extended this equation of motion by adding the spin torque terms. They showed that the Walker threshold current as well as the DW velocities strongly depends on the temperature. Furthermore, in their calculations, the velocities depend on the interplay between adiabatic and nonadiabatic spin torque contributions which might prove useful to experimentally extract their contributions. Furthermore, thermally activated hopping over an energy barrier cannot only lead to depinning but in the case of no or small injected currents, it can lead to hopping between two adjacent energy minima (pinning sites) [47]. This hopping can be analysed using rate theory [48] and for small injected currents, the change in the hopping rates can be used to determine the nonadiabaticity parameter $\beta$ [47].

\section{Experimental observation of current-induced DW motion}

We now come to the main part of this review and explore CIDM experimentally. Different techniques can be exploited for the measurements including magnetotransport, imaging by magnetic microscopy techniques and others. For an overview of techniques see for instance various chapters in $[8,49,50]$. For the experimental results we separate the systems into inplane magnetized materials (for instance Permalloy) and outof-plane magnetized materials (for instance $\mathrm{Co} / \mathrm{Pt}$ multilayers) that exhibit distinctly different DW structures.

\subsection{Current-induced DW motion in in-plane magnetized soft-magnetic wires}

We will here limit ourselves to head-to-head or tail-to-tail DWs in soft-magnetic permalloy $\left(\mathrm{Ni}_{80} \mathrm{Fe}_{20}\right)$ wires, as other materials (for instance polycrystalline $\mathrm{Ni}, \mathrm{Fe}$ or $\mathrm{Co}$ are expected to behave similarly albeit with more pinning). With the advent of advanced nano-lithography [51], which allows one to fabricate well-defined nanoscale magnetic wires, such nanostructures have become readily available. The samples are often prepared using electron beam lithography including deposition of permalloy/Au by molecular beam epitaxy on a naturally oxidized Si wafer and a double lift-off process [51].

Two DW types are generally found in these wires as shown in figure 2. In (a), a photoemission electron microscopy [52] image of a vortex head-to-head wall (VW) is shown together with a micromagnetic simulation where the spin structure is visualized together with the resulting contrast of the image. In $(c)$, a transverse wall is shown, which occurs in thinner and narrower structures (for details of the geometry-dependence of the wall spin structure see [8]). An example of experimentally observed DW displacements is shown in figures $2(b)$ and $(d)$. Here we see in $(b)$ that a vortex head-to-head DW is displaced by a current pulse with a high current density of $10^{12} \mathrm{~A} \mathrm{~m}^{-2}$ in the electron flow direction. In (d) we present the motion of a transverse DW.

Using ultra-short pulses with very fast rise times ( $<100 \mathrm{ps}$ ) we have recently imaged very fast DW motion with velocities $>100 \mathrm{~m} \mathrm{~s}^{-1}$ [53]. To create these DWs prior to the current injections, an external magnetic field is applied in a direction perpendicular to the wire. After reducing the field, this results in the DW being positioned in this direction in the centre of the wire. An X-ray magnetic circular dichroism photoemission electron microscopy (XPEEM) image of the initial configuration is presented in figure $3(a)$ (top wire). To visualize the spin structure, the inset in the lower left shows a micromagnetic simulation of a VW confined in a wire with the same contrast. Once the initial configuration is imaged, single current pulses are injected into the structure. The result of a series of injections is presented in figure 3 . Current pulses were injected between adjacent images (from top to bottom). After five injections, the DW is displaced by about $2 \mu \mathrm{m}$. figure $3(b)$ presents line scans showing the displacement and the transformation of the DW. We see that the right side of the DW continuously moves in the direction of electrons. 

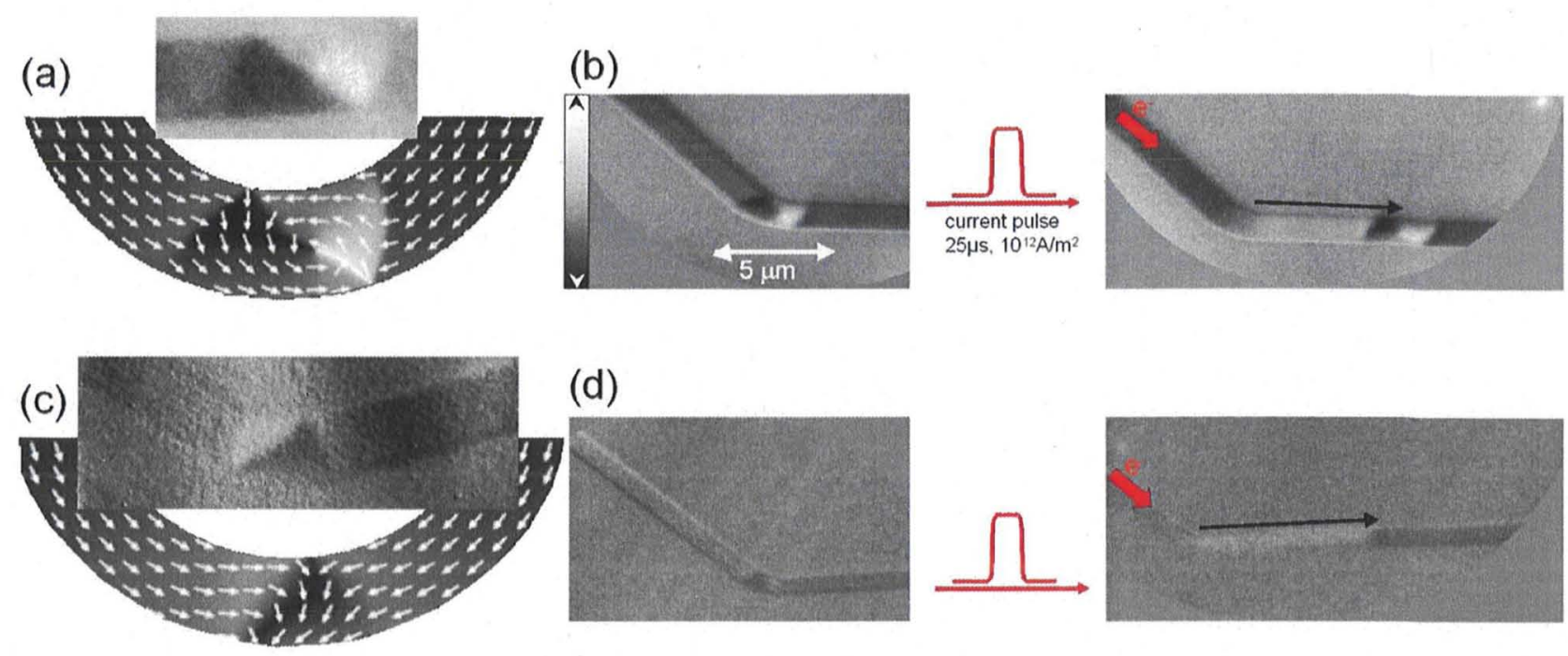

(d)
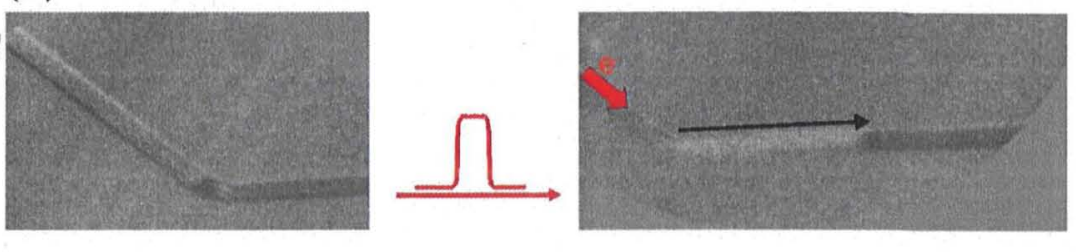

Figure 2. Photoemission electron microscopy images and corresponding micromagnetic simulations of $(a)$ a vortex head-to-head DW and $(c)$ a transverse wall. The shades of grey and the arrows indicate the magnetization directions. $(b)$ shows the displacement of a vortex wall by current injection in a $28 \mathrm{~nm}$ thick, $1 \mu \mathrm{m}$ wide permalloy wire. The grey scale bar shows the magnetic contrast direction for all the images. (d) shows the displacement of a transverse wall in a $7 \mathrm{~nm}$ thick and $500 \mathrm{~nm}$ wide wire. In both cases the wall spin structure stays the same after the displacement.

After the third current pulse, the left side does not move and it seems to be somehow pinned. The DW is therefore stretched, which results in an increase in the stray field energy. If this energy increase becomes larger than the energy required for a vortex core nucleation, it is energetically more favourable to transform to a double VW by vortex core nucleation. The new DW structure again displaces under current injection similar to the simple VW without changing its spin structure any more. The average displacement per current pulse is $400 \mathrm{~nm}$. With the measured pulse length of $3 \mathrm{~ns}$ this results in an average DW velocity of $v=130 \mathrm{~m} \mathrm{~s}^{-1}$, which is much larger than for long currents pulses $(>10 \mu \mathrm{s})$ shown in figure 2 . The fast wall motion as also observed by others for pulses with short rise times [54] can be explained by the combination of adiabatic and nonadiabatic torque that act initially before the adiabatic torque is balanced by the anisotropy [55]. Hence, by using pulses with fast rise times, that are shorter than the relaxation (damping) time for the DWs, a large torque can develop that aids depinning [55] and can lead to fast wall displacement in the viscous regime.

Experimentally, current-induced wall motion of these DWs has been studied by a large number of groups using various techniques (for an overview see citing papers and references in $[8,29,54,56-62])$.

Dynamic measurements of the velocity have been carried out by Hayashi et al for the case of a DW which is dynamically generated [62]. Starting with a DW at rest, wall motion as seen in figure $2[56,57]$ has been observed by a number of groups and for sufficiently high current densities, periodic wall transformations have been imaged [57,61]. From these observations of transformations one could deduce that the nonadiabaticity parameter $\beta$ does not equal the damping constant $\alpha$ [61]. A more quantitative measurement of $\beta$ [47] has recently shown that for wide transverse DWs where the magnetization gradients are small, $\beta$ is of the order of $\alpha$ thus pointing to spin relaxation as the dominating contribution to $\beta$, which arises from the same impurities that lead to viscous damping. For vortex walls a significantly larger value of $\beta$ was found and this can be attributed to nonadiabatic transport across the vortex core where particularly high magnetization gradients occur [47]. This means that there is a distinct dependence of the torque terms on the magnetization configuration and thus the torques can be tailored by appropriately engineering the spin structures.

In the next section we will go to high-anisotropy materials where high magnetization gradients occur in the narrow DWs.

\subsection{Current-induced DW motion in out-of-plane magnetized magnetic wires}

Most experimental studies on current-induced DW motion were carried out in soft-magnetic nanowires made of permalloy. This well-known material has the advantage of a DW less sensitive to pinning due to the large DW width $(\sim 100 \mathrm{~nm})$ and the long exchange length as well as the potentially high spin polarization. Experiments have, however, underlined serious limitations concerning the use of this material for the study of CIDM: high critical current densities leading to strong Joule heating, complex DW structures with uncontrolled DW transformation induced by current injection (see, for instance, figure 3(a)) [57,63] that leads to unreliable and stochastic DW displacements [64], and domain nucleation induced by current injection [65]. This limits the possibilities for a fundamental understanding of the spin-transfer effect in magnetic DWs but is also a serious issue for possible applications based on CIDM. Since 2004, a growing number of experiments were carried out on spin transfer in out-of-plane magnetized materials with a large perpendicular magnetic anisotropy. This class of materials combines several advantages over soft in-plane magnetized 

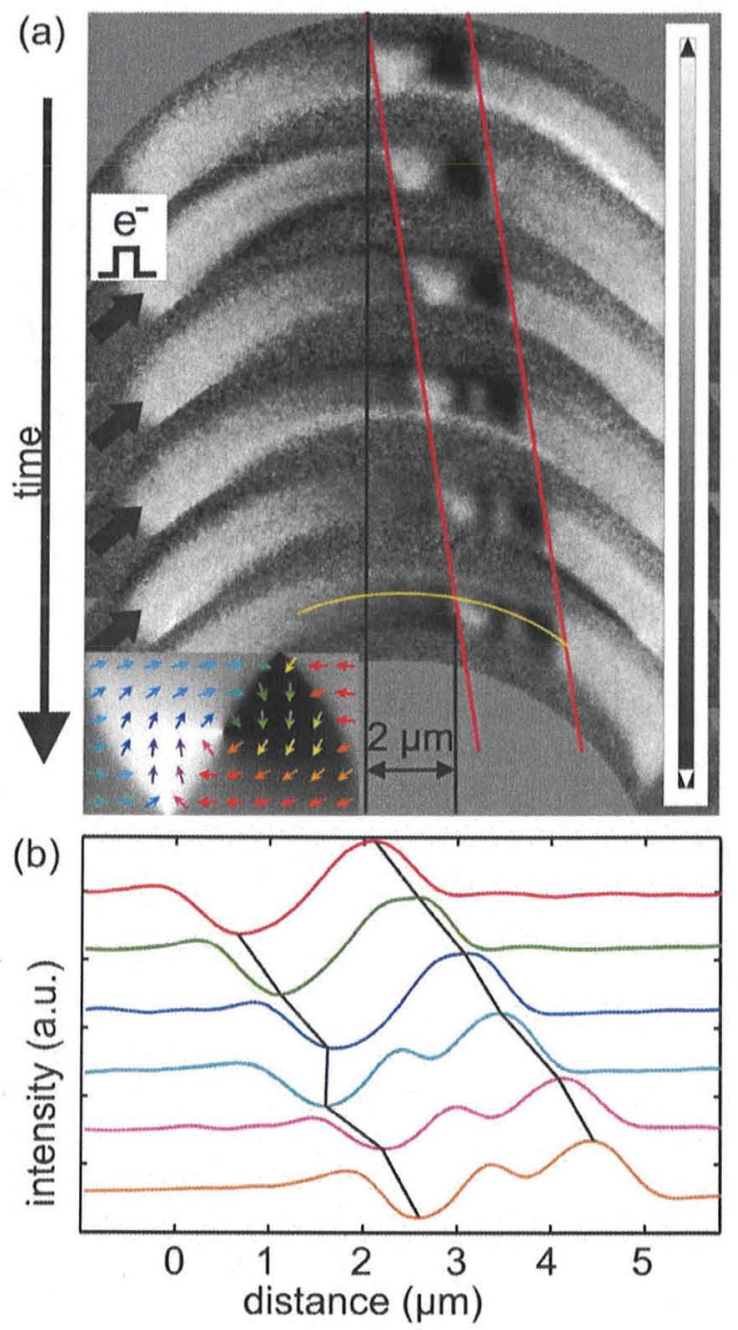

Figure 3. (From [53]) (a) Photoemission electron microscopy image series of a Py wire $(1000 \mathrm{~nm}$ wide, $40 \mathrm{~nm}$ thick with a $2 \mathrm{~nm}$ Au capping) containing a VW. The top image shows the configuration after the initialization by a short vertical field pulse. The inset in the lower left shows a micromagnetic simulation of a VW to help visualizing the DW structure. The XPEEM images were taken with vertical contrast (see grey scale bar at the right) and the simulation was adapted to this contrast. Current pulses were injected between adjacent images (from top to bottom). After five injections the DW is displaced by $2 \mu \mathrm{m}$, thus about $400 \mathrm{~nm}$ on average per current pulse. After the third pulse DW transformed to a double VW that continues moving. (b) Intensity line scans along the yellow curve indicated by the yellow line in $(a)$.

materials: narrow DWs typically below $10 \mathrm{~nm}$ with a simpler and more rigid internal Bloch/Néel DW structure; expected higher nonadiabaticity effects due to the higher magnetization gradients and high spin-orbit coupling leading to lower critical current densities and higher velocities; a large variety in the magnetic and transport properties of the available materials that allows one to study the dependence of spin-transfer effect on these parameters. For the prospect of high density magnetic memories based on CIDM, these advantages combined with a small DW width, i.e. small size of the magnetic bit, make these materials very attractive. In the following, we will restrict the discussion to metallic out-of-plane magnetized ferromagnetic materials with, for this cluster paper, a focus on our own work while citing the relevant other literature. In particular we will not address materials, such as diluted magnetic semiconductors. The reader can find more detailed information about this last topic in [66-68].

3.2.1. DWs in out-of-plane magnetized materials. Out-ofplane magnetized materials considered for CIDM experiments are mostly metallic thin films characterized by a strong uniaxial anisotropy oriented perpendicularly to the film plane. Two kinds of materials have been considered so far: ultrathin materials $(<1 \mathrm{~nm})$ where the anisotropy originates from the interface, such as in a $\mathrm{Pt} / \mathrm{Co} / \mathrm{Pt}$ multilayer, and thicker $(5-60 \mathrm{~nm})$ materials with a magnetocrystalline anisotropy that originates in the crystalline structure of the bulk. Due to the high anisotropy, the DW width is very small and typically ranges between 1 and $10 \mathrm{~nm}$. For ultra-thin magnetic films, the thicknesses are generally smaller than the exchange length or the DW width so that the magnetization can be considered as being uniform across the film $[49,69]$ and DWs exhibit a nearly perfect Bloch or Néel spin structure (see figures 4(a) and $(b))$.

In a nanowire geometry, the equilibrium DW configuration (Bloch or Néel) depends on the geometry of the sample [70]. One can define an effective out-of-plane anisotropy $K_{\text {eff }}=$ $K-N_{z} \mu_{0} M_{\mathrm{s}}^{2} / 2$ and a DW demagnetizing energy $K_{\mathrm{d}}=$ $\mu_{0} M_{\mathrm{s}}^{2}\left(N_{y}-N_{x}\right) / 2$, where $N_{x}, N_{y}$ and $N_{z}$ are the demagnetizing factors in the DW. $K_{\mathrm{d}}$ represents the magnetostatic energy difference between a Bloch and a Néel DW and the equilibrium DW configuration is a Bloch DW (respectively Néel DW) for $K_{\mathrm{d}}$ positive (respectively negative). For a very thin nanowire of thickness $t$ and width $w$, the DW cross section can be modelled as an ellipse and the demagnetizing factors can be approximated as $N_{y} \sim t /(t+$ $\pi \Delta)$ and $N_{x} \sim t /(t+w)$ [30,70]. In most experiments, the wire width (typically between 70 and $500 \mathrm{~nm}$ ) is large compared with the DW width so that $N_{y}>N_{x}$ and a Bloch DW is generally prevailing.

3.2.2. Simulation of CIDM in out-of-plane magnetized material. The recent interest in out-of-plane magnetized materials has stimulated the modelling and the micromagnetic simulation of the CIDM in these materials. The main features of the DW dynamics in a perfect nanowire are shown to be generally well described by a simple 1D model due to the simple DW Bloch structure. However, simulations also reveal important dynamical features due to the thin Bloch DW structure and the different magnetic properties of these materials [71-77].

Suzuki and Fukami et al [71-73] and Jung et al [75] studied the dependence of the critical current density on the nanowire geometry (width and thickness). Using both 1D model and micromagnetic simulation, Jung et al [75] showed that for narrow (typically below $100 \mathrm{~nm})$ and thin $(<10 \mathrm{~nm})$ nanowires, the critical current density $J_{\mathrm{c}}$ does not depend on the pinning strength as well as on the nonadiabaticity factor $\beta$ and reaches a minimal value for a certain geometry (width-thickness) of the wire. For this critical geometry, the 


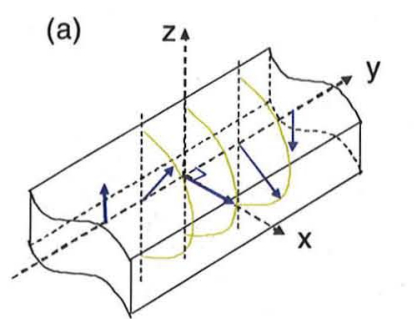

Bloch DW

(c)

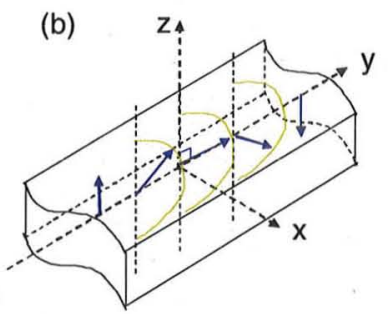

Néel DW

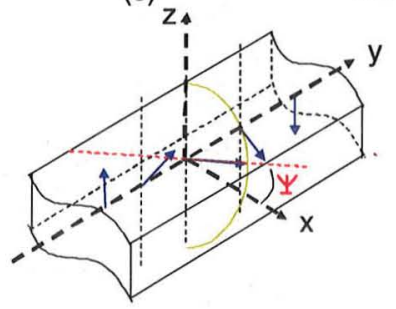

Figure 4. $(a)-(b)$ Schematic representation of a Bloch $(\psi=0)(a)$ and a Néel $(\psi=\pi / 2) \mathrm{DW}(b)$. (c) DW with a non zero internal angle $\psi$.

demagnetizing energy $K_{d}$ changes sign and the DW switches from a Bloch to a Néel structure. The intrinsic critical current associated with the adiabatic torque proportional to $K_{\mathrm{d}}$ thus becomes very low $\left(J_{\mathrm{c}}<10^{10} \mathrm{~A} \mathrm{~m}^{-2}\right)$ (in an ideal geometry it would vanish as the anisotropy barrier goes to zero as also for round wires with shape anisotropy [78]) and controls the depinning process. As a consequence, the nonadiabatic spin torque term $\beta$ and extrinsic pinning effect play a little role on the depinning. The geometry for minimal $J_{\mathrm{c}}$ is obtained for thick enough and very narrow wires: for a $10 \mathrm{~nm}$ thick film, Jung et al [75] found the critical current density to be minimal for $w \approx 70 \mathrm{~nm}$, with the exact dimensions depending critically on the magnetic parameters.

The possibility to combine low intrinsic critical current density and high pinning forces can also be exploited to generate new steady dynamical states. Above the intrinsic current density, the DW enters a precession regime with a periodic change of the internal DW structure from Bloch to Néel associated with an oscillation of the internal angle $\psi$ with time (the so-called Walker regime). If pinning is not very high, this leads to DW depinning followed by DW propagation with a velocity oscillating in time $[79,80]$. However, if the pinning is strong enough, the DW stays in the pinning potential well and a steady oscillation of the DW in the well can be obtained. This actually corresponds to the high pinning case of Tatara et al [14], with a pinning field $H_{\mathrm{p}}$ larger than $H_{k} / \alpha$, with $H_{k}=2 K_{\mathrm{d}} /\left(\mu_{0} M_{\mathrm{s}}\right)$, the DW demagnetizing field. Outof-plane magnetized nanowires are well suited to obtain small $H_{k} / \alpha$ as they are generally characterized by high values of $\alpha$ (typically higher than 0.1 in $(\mathrm{Co} / \mathrm{Pt})_{n}$ multilayers [81]) and a very low value of $H_{k}$ can be obtained by playing on the wire geometry. We have demonstrated the validity of this idea using micromagnetic simulations [82]. We have considered a Bloch DW pinned in a $16 \mathrm{~nm}$ wide notch patterned in a $7 \mathrm{~nm}$ thick wire. The magnetic parameters considered are typical of $(\mathrm{Co} / \mathrm{Pt})_{n}$ multilayers and only the effect of the adiabatic torque is taken into account $(\beta=0)$. This geometry corresponds (a)
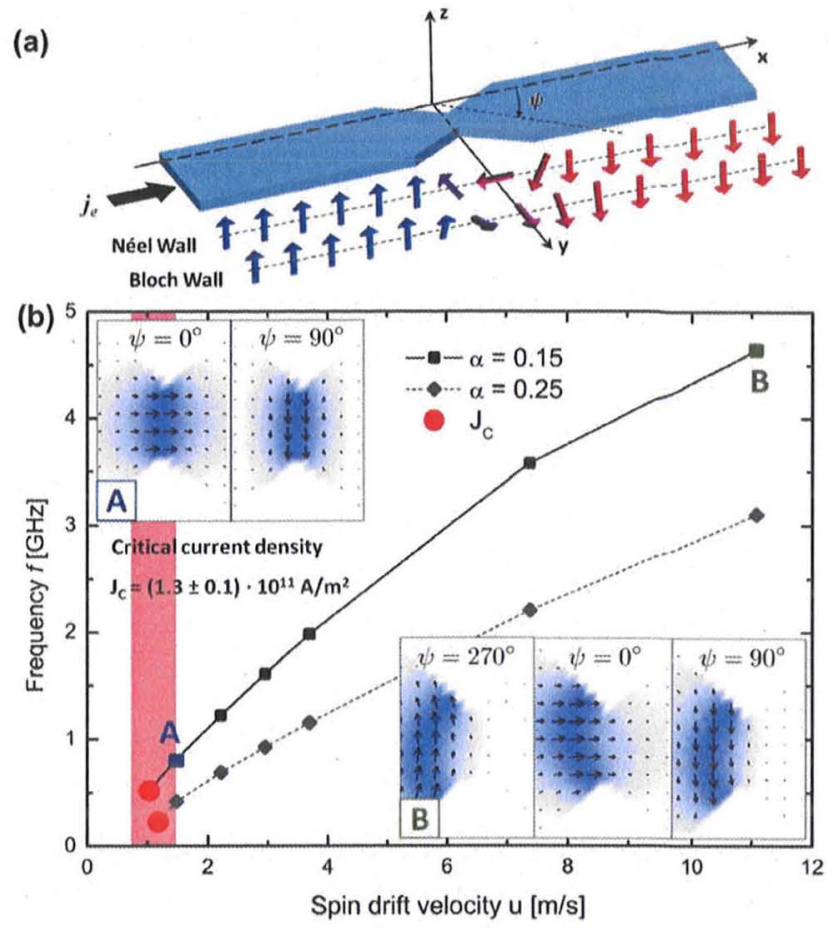

Figure 5. (From [82]). (a) Schematic illustration of the geometrically confined structure. The arrows represent the magnetization configuration inside the structure, which can be either a Bloch or a Néel DW. $(b)$ DW oscillation frequency $f$ as a function of the injected spin current drift velocity $u$ for constant

$\alpha=(0.15,0.25)$. (A) The DW profile is symmetric under rotation for low current density $j_{e}=1.79 \times 10^{11} \mathrm{~A} \mathrm{~m}^{-2}$. (B) At a high current density $j_{e}=1.34 \times 10^{12} \mathrm{~A} \mathrm{~m}^{-2}$, the DW shows asymmetric oscillations, this leads to a nonharmonic behaviour.

actually to a minimal value of $H_{k}$ and a corresponding critical current density of $J_{\mathrm{c}}=1.34 \times 10^{11} \mathrm{~A} \mathrm{~m}^{-2}$. Micromagnetic simulations show that for $J$ above $J_{\mathrm{c}}$, the DW stays pinned in the potential well and periodically oscillates between a Bloch and a Néel structure. The average frequency $\langle\dot{\psi}\rangle$ scales approximately linearly with the spin current drift velocity $u=J P \mu_{\mathrm{B}} / e M_{\mathrm{S}}$ (see figure 5) in agreement with a simple $1 \mathrm{D}$ model that predicts $\langle\dot{\psi}\rangle=-u / \alpha \Delta$. This simple concept of localized DW steady-state oscillator opens an interesting way for current controlled tunable nanoscale microwave oscillators that can work at zero external magnetic field.

\subsubsection{Experimental examples of current-induced DW motion in out-of-plane magnetized wires.}

Experimental considerations. Due to the particular geometry of the wires and the anisotropy, respectively, the magnetization direction, other effects beyond the spin torque effect can occur when injecting current in out-of-plane magnetized nanowires. This means that particular cares is necessary to separate these effects when interpreting the data.

Joule heating. The temperature increase due to the Joule heating can strongly affect the DW dynamics [83-88]. For a given current density, the heating amplitude depends on 

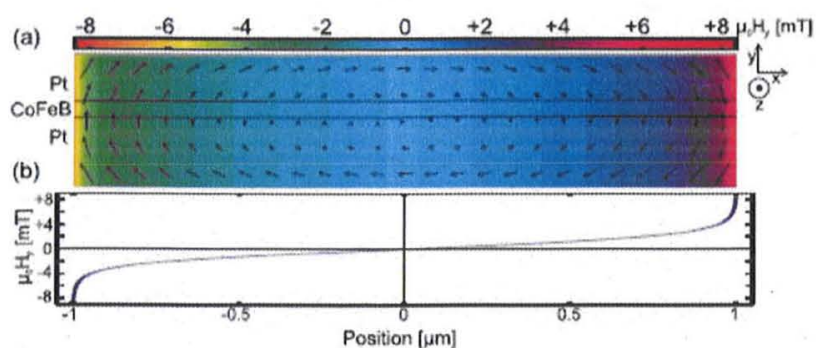

Figure 6. (From [93]). Cross-section of a wire with the calculated distribution of the Oersted field. The current $\left(10^{12} \mathrm{~A} \mathrm{~m}^{-2}\right.$ flows homogeneously in the wire in the $-z$ direction. The wire dimensions are $5.6 \mathrm{~nm} \times 2 \mu \mathrm{m}$ ( $x$ and $y$ are plotted at different scales). The out-of-plane component of the Oersted field $H_{y}$ is plotted in colour. (b) $H_{y}$ as a function of the lateral position $x$ in the wire.

the material and the geometry of the wire as well as on the metallic contacts and the nature of the substrate where heat is dissipated [84, 85]. In typical CIDM experiment, heating can reach up to several hundred $\mathrm{K}$ for high current densities ( $\sim 10^{11}-10^{12} \mathrm{~A} \mathrm{~m}^{-2}$ depending on experiments) [83, 87-90]. In out-of-plane magnetized materials with strong DW pinning, the DW dynamics is dominated by thermal activation regime over a large range of current and field values. In this regime, the DW dynamics is particularly sensitive to the temperature increase due to Joule heating that helps the DW to overcome the pinning energy barrier. This is particularly true in the case of current-induced DW motion or depinning experiments in the presence of an external magnetic field that lowers the pinning energy barrier $[87,91]$. Even if Joule heating cannot be suppressed, its amplitude can be significantly reduced using magnetic materials with small resistivity, thin films, and choosing a substrate with high thermal conductivity $[84,85]$, such as Si or diamond [92]. Another approach is to cover the wire with a high thermal conductivity but electrically insulating layer to play the role of a heat sink, such as an AIN capping [87].

Oersted field effect. The concentric Oersted field can play a significant role for the magnetization dynamics in out-ofplane magnetized nanowire. Figure 6 shows the simulated distribution of the out-of-plane component of the Oersted field in a nanowire [93] which shows a rapid increase in the field amplitude as one approaches the wire edges. Actually, the maximum Oersted field on the sides $H_{z}$ depends on the width $w$ and the thickness $t$ of the wire and can be approximated as $H_{z}=J t(3+2 \ln (w / t)) / 4 \pi$ for a thin conductor $(t \ll w)[94]$ and is thus larger for wide and thick nanowires. For a typical $200 \mathrm{~nm}$ wide $5 \mathrm{~nm}$ thick wire, the resulting Oersted field is about $5 \mathrm{mT}$ for $J=1 \times 10^{12} \mathrm{~A} \mathrm{~m}^{-2}$. Although the net force on the complete DW is zero, such an Oersted field can bend the DW at the edges and lead to an asymmetric DW motion [94]. Experimentally, the influence of the Oersted field on the DW depinning and propagation can be identified by comparing the DW dynamics for domain configurations with opposite magnetization [95].

In wide nanowires in soft out-of-plane magnetized materials, the Oersted field can also have some unexpected effects on the domain configuration in the nanowires.

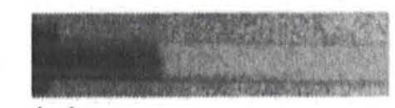

(a)

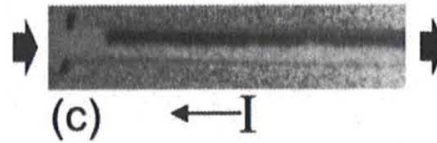

(c)

Figure 7. (From [93]). XPEEM magnetic images of a $2 \mu \mathrm{m}$ wide $\mathrm{Pt} / \mathrm{CoFeB} / \mathrm{Pt}$ wire. A white contrast corresponds to the magnetization pointed up and a black contrast to the magnetization pointing down. (a) A DW is shown in the wire. (b) After the injection of a current pulse $J=1.0 \times 10^{12} \mathrm{~A} \mathrm{~m}^{-2}$ for $25 \mu \mathrm{s}$, direction indicated by the arrows in the wire, the original DW structure disappears and a long DW parallel to the wire is created. When the current direction is reversed, the magnetization in the domain also reverses $((c)$ and $(d))$.

Figure 7(a) shows a magnetic image of a DW in a $2 \mu \mathrm{m}$ wide nanowire patterned in out-of-plane magnetized $\mathrm{Pt} / \mathrm{CoFeB}(0.6 \mathrm{~nm}) / \mathrm{Pt}$. This comparably soft material is characterized by a relatively low coercive ficld $(\sim \mathrm{mT})$. When injecting a high current density in the nanowire $\left(\sim 10^{12} \mathrm{~A} \mathrm{~m}^{-2}\right)$, a new domain structure appears with two domains with opposite magnetization separated by a DW aligned along the wire (see figure $7(b)$ ). The appearance of this domain structure is attributed to the combined effect of the Oersted field and the reduction of the magnetostatic energy (closure of flux line). Interestingly, when injecting successive current pulses of opposite polarities, the magnetization in the DW can be reversed back and forth by the sole effect of the Oersted field (see figures 7(c) and $(d)$. This underlines the high Oersted field generated in these wide wires, which was estimated to reach $8 \mathrm{mT}$ on the edges for $J=1 \times 10^{12} \mathrm{~A} \mathrm{~m}^{-2}$.

Experiments to determine the spin torque terms in out-of-plane magnetized wires. A large number of experiments on currentinduced DW motion in out-of-plane magnetized structures were devoted to the characterization of the highly debated nonadiabatic torque. Most of these experiments were carried out in the presence of an external magnetic field in addition to the injected current. Indeed, the effect of the nonadiabatic torque on the DW dynamics is predicted to be equivalent to an external magnetic field $[13,41,77]$ so that for instance the relative changes induced by the current in the depinning field allows one to measure nonadiabatic effects.

Variation of the depinning field with current injection. In most experiments $[74,87,95,96,97]$, the depinning field $H_{\text {dep }}$ strongly decreases as current is injected and for sufficiently high current densities a linear dependence is found. Importantly, the slope $\epsilon=\mu_{0} \Delta H_{\mathrm{dep}} / \Delta J$ of the curve $H_{\mathrm{dep}}(J)$, generally called 'efficiency', is much higher compared with what is observed in permalloy $[86,87]$.

As an example, we have studied the variation of the depinning field when injecting $50 \mu$ s current pulses in an out-of-plane magnetized $(\mathrm{Pt} / \mathrm{Co}(0.6 \mathrm{~nm}))_{3} / \mathrm{Pt}$ Hall cross with $540 \mathrm{~nm}$ lateral dimensions at several temperatures. At constant cryostat temperature, a strong decrease in the depinning field 

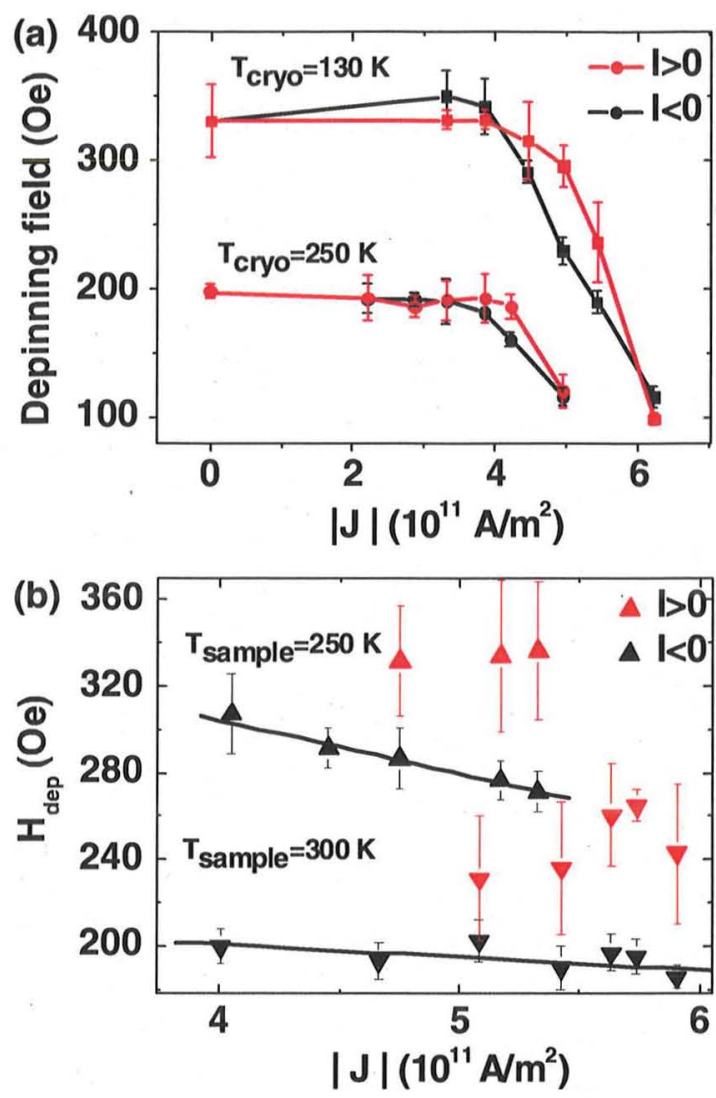

Figure 8. Depinning field as a function of $|J|$ for $(a)$ a constant cryostat temperature of $T_{\text {cryo }}=130 \mathrm{~K}$ (squares) and $T_{\text {cryo }}=250 \mathrm{~K}$ (circles) and (b) a constant sample temperature of $T_{\text {sample }}=250 \mathrm{~K}$ (up triangles) and $T_{\text {sample }}=300 \mathrm{~K}$ (down triangles).In $(b)$, the black lines are a linear fit of the data.

is observed for both current polarities when injecting current densities higher than $4 \times 10^{11} \mathrm{~A} \mathrm{~m}^{-2}$ although the depinning field is always higher for one current polarity compared with the other one (see figure 8(a)). From the slope of the curve for $T_{\text {cryo }}=130 \mathrm{~K}$, one obtains a high effective efficiency $\epsilon \sim 10^{-13} \mathrm{~T} \mathrm{~m}^{2} \mathrm{~A}^{-1}$. However, the weak dependence of the depinning field on the current polarity clearly suggests an important contribution of the temperature rise due to Joule heating, that was measured to be about $200 \mathrm{~K}$ for the maximum injected current.

To extract the spin torque contribution, we carried out the same depinning experiment but at a constant sample temperature by playing on the external temperature and the amplitude of the injected current. Figure $8(b)$ shows the resulting depinning field versus current density curve for two different sample temperatures of $T_{\text {sample }}=250 \mathrm{~K}$ and $T_{\text {sample }}=300 \mathrm{~K}$. We observe now a clearly different behaviour: the depinning field decreases linearly for one current polarity (current helps the DW depinning) whereas it is approximately constant or slightly increases for the other current polarity (current hinders DW depinning). From the slope of the current polarity for negative current that helps the depinning, one can derive a real efficiency $\epsilon=2.5 \pm 0.35 \times 10^{-14} \mathrm{~T} \mathrm{~m}^{2} \mathrm{~A}^{-1}$ for $T_{\text {sample }}=250 \mathrm{~K}$ and $\epsilon=6 \pm 1.5 \times 10^{-15} \mathrm{~T} \mathrm{~m}^{2} \mathrm{~A}^{-1}$ for $T_{\text {sample }}=$
$300 \mathrm{~K}$. To analyse the results, we carried out simulations of the DW dynamics using the 1D model with thermal activation (see section 2). We assume a pinning potential $V_{\text {pin }}(q)$ such that $V_{\text {pin }}$ is quadratic with the position $q$ in the potential well and constant outside. Our simulations show that only the nonadiabatic torque can change the depinning field. Actually, the adiabatic torque tilts the DW internal angle $\psi$ but does not change the equilibrium position of the DW in the potential well due to the damping. In contrast, the nonadiabatic torque modifies the potential profile as well as the DW position and thus acts on the pinning energy barrier. In the 1D model, the nonadiabatic torque acts on the DW as an effective field $\mu_{0} H_{I}=\epsilon I$ with [13]

$$
\epsilon=\beta P \hbar /\left(2 e M_{\mathrm{s}} \Delta\right) .
$$

From the experimental efficiency, one obtains $\beta=1.45 \pm$ 0.25 from the $T=250 \mathrm{~K}$ experiment and $\beta=0.35 \pm 0.08$ from the $T=300 \mathrm{~K}$ experiments to be compared with the damping parameter $\alpha \sim 0.15$ in our film. Note that these values are rough estimations as the spin polarization is not known and assumed to be similar to the one in Co $(P=0.46)$. This value is, however, in the range of the spin polarizations extracted by Cormier et al [88] in $\mathrm{Pt} / \mathrm{Co}(0.5 \mathrm{~nm}) / \mathrm{Pt}$ from CIP GMR data $(P \approx 0.35)$ and by Aziz et al [98] by DW resistance measurement $(P \approx 0.7)$. Finally, the model does not explain the difference in the efficiency between both temperatures.

We also carried out complementary experiments at constant cryostat temperature $(T=100 \mathrm{~K})$ to characterize the contribution of the Oersted field in the DW depinning. This is done by comparing the depinning field for opposite domain configuration around the DW. We find a negligible contribution of the Oersted field much smaller than that of the nonadiabatic torque [95].

Several authors studied in more details the dependence of $J_{\mathrm{c}}$ on $H_{\mathrm{dep}}$ in similar materials. Ravelosona et al [99] and $\mathrm{Li}$ et al [100] reported in $0.2-1 \mu \mathrm{m}$ wide $\mathrm{Pt} / \mathrm{Co}$ wires and $8 \mu \mathrm{m}$ wide TbFeCo nanowire an approximately linear scaling of $J_{c}$ with the pinning field which is consistent with a depinning controlled by the nonadiabatic torque. However, a different scaling was reported in narrow (70-240 nm wide) $(\mathrm{Co} / \mathrm{Ni})_{4,5}$ wire $[74,101-103]$ where the critical current was found to be independent of external pinning nor on the external field. Koyama et al [103] also studied the dependence of $J_{\mathrm{c}}$ on the wire width $w$ for $w$ ranging between 40 and $300 \mathrm{~nm}$. They observe a minimum in the critical current density for a given width of the wire corresponding to the transition from a Bloch to a Néel DW where the DW demagnetizing field is minimum. This behaviour is clearly consistent with an 'intrinsic pinning' behaviour where the depinning process is driven by the adiabatic torque in this case. Indeed, as discussed in section 3.2.2, the critical current associated with the adiabatic torque can be lower than the one associated with the nonadiabatic torque for narrow wire due to the small DW demagnetizing field. The nonadiabatic torque plays thus a minor role in these experiments. 
Table 1. Summary of results from CIDM in the presence of an external magnetic field. Type of experiment: D: dependence of the depinning magnetic field with the injected current; I: dependence of the depinning time with the injected current; Di: displacement of the DW with the injected current and external magnetic field. Cr: dependence of the DW velocity on current and field in the thermally activated creep regime. (1): This best estimate value was corrected compared with the published one to take into account different definitions of $\epsilon$ and $\beta$. (2) Rough estimate assuming typical parameter for the material.

\begin{tabular}{|c|c|c|c|c|c|c|c|}
\hline Material & $\Delta(\mathrm{nm})$ & $\epsilon\left(10^{-14} \mathrm{~T} \mathrm{~m}^{2} \mathrm{~A}^{-1}\right)$ & $\beta$ & $\beta_{\min }$ & $\alpha$ & Type of exp. & Ref \\
\hline $\begin{array}{l}{[\mathrm{Co}(0.5) / \mathrm{Pt}]_{4} /} \\
\mathrm{Co}(0.5) \mathrm{Cu} / \mathrm{Co}(0.5) / \mathrm{Pt}(\mathrm{SV})\end{array}$ & 10 & 17 & & 7.6 & & I & {$[96]$} \\
\hline $\mathrm{Pt} / \mathrm{Co}(0.6) / \mathrm{Pt}$ & 4.2 & $<0.2$ & $<0.05^{(1)}$ & 0.029 & & $\mathrm{Di}$ & [106] \\
\hline $\mathrm{Pt} / \mathrm{Co}(0.3 \mathrm{~nm}) / \mathrm{Pt}$ & $4.5^{(2)}$ & $1.6 \pm 0.1$ & & 0.34 & & $\mathrm{Cr}$ & [104] \\
\hline $\mathrm{Pt} /[\mathrm{Co}(0.6) / \mathrm{Pt}]_{3}$ & 6.3 & $0.6 \pm 0.152 .5 \pm 0.35$ & $0.35 \pm 0.081 .45 \pm 0.25$ & $0.16-0.67$ & 0.15 & $\mathrm{D}$ & [87] \\
\hline $\mathrm{Pt} /[\mathrm{Co}(0.5) / \mathrm{Pt}]_{2}$ & $4^{(2)}$ & $3.6 \pm 0.6$ & $0.7 \pm 0.1$ & 0.49 & & $\mathrm{Cr}$ & [105] \\
\hline $\mathrm{Pt} / \mathrm{Co} / \mathrm{AlO}_{x}$ & 4.2 & 8 & $1.9^{(1)}$ & 1.16 & 0.5 & $\mathrm{Di}$ & [106] \\
\hline$(\mathrm{Co} / \mathrm{Ni})_{4} / \mathrm{Co} / \mathrm{Pd}(\mathrm{SV})$ & 10 & 1.5 & $0.022 \pm 0.002$ & 0.21 & 0.032 & I, D & [91] \\
\hline $\mathrm{SrRuO}_{3}$ & 1 & 100 & & 0.48 & & $\mathrm{D}$ & [107] \\
\hline $\mathrm{FePt}(\mathrm{SV})$ & 1 & 30 & $0.06 \pm 0.03$ & 0.9 & 0.1 & I, D & [91] \\
\hline
\end{tabular}

Experiments in further materials. The nonadiabatic factor was characterized by several groups in other materials such as ultra-thin magnetic films $\left(\mathrm{Pt} / \mathrm{Co} / \mathrm{AlO}_{x}, \mathrm{Pt} /(\mathrm{Co} / \mathrm{Pt})_{n},(\mathrm{Co} / \mathrm{Ni})_{n}\right.$ multilayers) and thicker films with a strong volume anisotropy $\left(\mathrm{SrRuO}_{3}, \mathrm{FePt}\right)$. This was done using different techniques: DW creep in the presence of current and field [104, 105], small displacements of a DW in a pinning potential driven by current and field [106], waiting-time measurement of the DW depinning at constant current and field [91]. Table 1 summarizes these different results. The first column lists the different values of the experimental efficiency. The second column list best estimates of $\beta$ of the authors from their experimental data. $\beta$ is estimated from a current/ field equivalence using equation (5) [87, 104-107] or from the linear variation with current of the pinning barrier energy $[42,91]$. Note that $\beta$ is directly proportional to the current spin polarization $P$, which is subject to large uncertainty. Furthermore, the two different methods can lead to very different estimates of $\beta$ in the same material [91]. To take into account these bias, we also computed $\beta$ from the efficiency $\epsilon$ using the current/field equivalence (equation (5)) and assuming $P=1$. This value $\beta_{\min }$ corresponds actually to a lower bound for $\beta$ derived from the current/field equivalence.

Discussion of the experimental results. Table 1 shows a large dispersion of $\epsilon$ and $\beta_{\min }$ for different materials and experiments. A striking example is the large difference for $\epsilon$ and $\beta$ for $\mathrm{Pt} / \mathrm{Co}(0.3 \mathrm{~nm}) / \mathrm{Pt}$ in [104] and $\mathrm{Pt} / \mathrm{Co}(0.6 \mathrm{~nm}) / \mathrm{Pt}$ for [106]. In addition to the variation in the spin polarization for the different materials, this suggests that $\beta$ depends also strongly on the exact materials composition and structure.

Interestingly, despite large variations in $\epsilon, \beta_{\min }$ typically ranges between 0.2 and 1 , which is much higher than the values of $\beta$ estimated from measurements in permalloy. There is currently no consensus to explain such a high nonadiabaticity in these materials and this remains an open question. One can, however, invoke several different approaches for an explanation. One relies on the high spin orbit coupling in these materials with strong uniaxial anisotropy. Actually, strong nonadiabaticity was predicted in systems with high intrinsic spin-orbit coupling, such as in 2D magnetic gas with a Rashba spin-orbit interaction or (Ga,Mn)As [108-110]. This scheme seems to support the enhancement of the nonadiabaticity observed in $\mathrm{Pt} / \mathrm{Co} / \mathrm{Al}_{2} \mathrm{O}_{3}$ where a strong Rashba spin-orbit coupling was recently identified [111]. Momentum transfer expected for narrow DW is another possibility. However, this mechanism is expected to be relevant for DWs with widths of the order of the Fermi wavelength $(\sim 1 \mathrm{~nm})$ or the Larmor precession length (a few $\mathrm{nm}$ ) $[14,23]$ which is not completely the case for standard out-of-plane magnetized multilayers with larger DW widths $(\sim 5-10 \mathrm{~nm})$. The condition seems to hold though in $\mathrm{FePt}$ and $\mathrm{SrRuO}_{3}$ with very narrow DW $(\sim 1 \mathrm{~nm})^{5}$, explaining the apparent higher efficiency and nonadiabaticity. Furthermore, there seems to be no clear correlation between the measured $\beta_{\min }$ and $\alpha$ and in general, $\beta_{\min }$, which is a minimum boundary for $\beta$, is higher than $\alpha$, which seems to contradict recent theory predicting $\alpha$ of the order of $\beta$ [113]. However, one should consider that except for [106], the value of $\alpha$ was obtained from FMR or magnetic relaxation experiments on thin film which might differ from the one extracted from DW velocity measurements and effectively felt by the DW [114]. Actually, recent theories predict an enhancement of the damping parameter in the case of narrow DW [115].

\section{Conclusions}

In conclusion we have reviewed different concepts for DW motion based on spin-polarized currents. In soft inplane magnetized nanowires, such currents allow for DW displacement due to the spin-transfer torque effect and in this case all walls (for instance head-to-head and tail-to-tail in in-plane magnetized materials) move in the direction of the electron flow. The exact details of the wall motion depend on the interplay of the acting torques (adiabatic and nonadiabatic spin torque terms) with the intrinsic and shape anisotropies. For sufficiently high current densities, the torques lead to a deformation and even a transformation of the DW spin structures (Walker breakdown), which significantly influences the wall velocity. In soft in-plane magnetized wires this leads to transformations between transverse and vortex DWs. Recent experiments in out-of-plane magnetized wires with narrow Bloch DW have shown that the current acts on the DW similarly

5 Although the role of scattering may have to be considered [112]. 
to a strong external field, which indicates high nonadiabatic effects. Experimentally we observe this effect pointing to a nonadiabaticity constant $\beta$, which is not equal to the damping constant $\alpha$. By measuring the depinning process under fields and current in high-anisotropy materials with well-defined narrow DWs we show how absolute values of this torque term can be deduced and that much larger values are found than for in-plane magnetized wires with wide DWs. These materials are very promising for memory devices based on CIDM as lower critical current density combined with high pinning force (bit stability) and high DW velocity can be expected from the higher nonadiabacity. A future challenge is now to decrease the strong pinning due to the natural defects in these materials that leads to high critical current density and makes it hard to accurately control the position of the DW in the track. A possible route is the use of softer or epitaxial out-of-plane material. From a fundamental point of view, the origin of the higher nonadiabaticity in these materials still remains an important open question even if the high spin-orbit coupling is a possible explanation. Answers may be found in further studies of nonadiabatic effects by taking advantage of the large diversity of available materials.

\section{Acknowledgments}

The authors acknowledge support by the DFG (KL1811 and SFB 767), the E.U. (Human Resources and Mobility Programme and SPINSWITCH MRTN-CT-2006-035327), the European Research Council through a Starting Independent Researcher Grant (ERC-2007-StG 208162), the EU (MAGWIRE FP7-ICT-2009-5 257707) the Swiss National Science Foundation and the Samsung Advanced Institute of Technology. GM would like to thank A Thiaville and J-Y Chauleau for fruitful discussions.

\section{References}

[1] McFadyen I R, Fullerton E E and Carey M J 2006 State-of-the-art magnetic hard disk drives MRS Bull. 31379

[2] Dee R H 2006 Magnetic tape: the challenge of reaching hard-disk-drive data densities on flexible media MRS Bull. 31404

[3] Tehrani S, Slaughter J M, Chen E, Durlam M, Shi J and DeHerrera M 1999 Progress and outlook for mram technology IEEE Trans. Magn. 352814

[4] Parkin S S P 2004 US Patent 6,834,005 and patent application $10 / 984,055$

[5] Hayashi M, Thomas L, Moriya R, Rettner C and Parkin S S P 2008 Current-controlled magnetic domain-wall nanowire shift register Science 320209

[6] Cowburn R, Petit D, Read D and Petracic O 2007 Patent WO 2007/132174A1

[7] Parkin S S P 2006 Spin-polarized current in spin valves and magnetic tunnel junctions MRS Bull. 31389

[8] Kläui M 2008 Head-to-head domain walls in magnetic nanostructures J. Phys.: Condens. Matter 20313001

[9] Tatara G, Kohno H and Shibata J 2008 Microscopic approach to current-driven domain wall dynamics Phys. Rep. $468213-301$

[10] Berger L 1978 Low-field magnetoresistance and domain drag in ferromagnets J. Appl. Phys. $\mathbf{4 9} 2156$
[11] Berger L 1984 Exchange interaction between ferromagnetic domain wall and electric current in very thin metallic films J. Appl. Phys. 551954

[12] Zhang S and Li Z 2004 Roles of nonequilibrium conduction electrons on the magnetization dynamics of ferromagnets Phys. Rev. Lett. 93127204

[13] Thiaville A, Nakatani Y, Miltat J and Suzuki Y 2005 Micromagnetic understanding of current-driven domain wall motion in patterned nanowires Europhys. Lett. 69 990-6

[14] Tatara G and Kohno H 2004 Theory of current-driven domain wall motion: spin transfer versus momentum transfer Phys. Rev. Lett. 92086601

[15] Tatara G, Takayama T, Kohno H, Shibata J, Nakatani Y and Fukuyama H 2006 Threshold current of domain wall motion under extrinsic pinning, $\beta$-term and non-adiabaticity J. Phys. Soc. Japan 75064708

[16] Tserkovnyak Y, Skadsem H J, Brataas A and Bauer G E W 2006 Current-induced magnetization dynamics in disordered itinerant ferromagnets Phys. Rev. B 74144405

[17] Tserkovnyak Y, Brataas A and Bauer G E W 2008 Theory of current-driven magnetization dynamics in inhomogeneous ferromagnets J. Magn. Magn. Mater. 320 1282-92

[18] Vanhaverbeke A and Viret M 2007 Simple model of current-induced spin torque in domain walls Phys. Rev. B 75024411

[19] Barnes S E and Maekawa S 2005 Current-Spin coupling for ferromagnetic domain walls in fine wires Phys. Rev. Lett. 95107204

[20] Kohno H, Tatara G and Shibata J 2006 Microscopic calculation of spin torques in disordered ferromagnets J. Phys. Soc. Japan 75113706

[21] Duine R A, Núñez A S, Sinova J and MacDonald A H 2007 Functional keldysh theory of spin torques Phys. Rev. B 75214420

[22] Waintal X and Viret M 2004 Current-induced distortion of a magnetic domain wall Europhys. Lett. 65 427-33

[23] Xiao J, Zangwill A and Stiles M D 2006 Spin-transfer torque for continuously variable magnetization Phys. Rev. B 73054428

[24] Ohe J I and Kramer B 2006 Dynamics of a domain wall and spin-wave excitations driven by a mesoscopic current Phys. Rev. Lett. 96027204

[25] Thorwart M and Egger R 2007 Current-induced nonadiabatic spin torques and domain-wall motion with spin relaxation in a ferromagnetic metallic wire Phys. Rev. B 76214418

[26] Thiaville A, Nakatani Y, Piéchon F, Miltat J and Ono T 2007 Transient domain wall displacement under spin-polarized current pulses Eur. Phys. J. B 60 5-27

[27] Thiaville A and Nakatani Y 2009 Micromagnetics of domain-wall dynamics in soft nanostrips Nanomagnetism and Spintronics (Amsterdam: Elsevier) pp 231-76

[28] Thiaville A and Nakatani Y 2006 Domain Wall Dynamics in Nanowires and Nanostrips (Berlin: Springer)

[29] Thomas L, Hayashi M, Jiang X, Moriya R, Rettner C and Parkin S S P 2006 Oscillatory dependence of current-driven magnetic domain wall motion on current pulse length Nature 443 197-200

[30] Porter D G and Donahue M J 2004 Velocity of transverse domain wall motion along thin, narrow strips $J$. Appl. Phys. 956729

[31] Schryer N L and Walker L R 1974 The motion of $180^{\circ}$ domain walls in uniform dc magnetic fields J. Appl. Phys. 45 5406-21

[32] Thiaville A 2004 Domain wall motion by spin-polarized current: a micromagnetic study J. Appl. Phys. 957049

[33] Chauleau J-Y, Weil R, Thiaville A and Miltat J 2010 Magnetic domain walls displacement: automotion versus spin-transfer torque Phys. Rev. B 82214414 
[34] Tatara G, Vernier N and Ferré J 2005 Universality of thermally assisted magnetic domain-wall motion under spin torque Appl. Phys. Lett. 86252509

[35] Duine R A, Núñez A S and MacDonald A H 2007 Thermally assisted current-driven domain-wall motion Phys. Rev. Lett. 98056605

[36] Martinez E, Lopez-Diaz L, Alejos O, Torres L and Tristan C 2007 Thermal effects on domain wall depinning from a single notch Phys. Rev. Lett. 98267202

[37] Martinez E, Lopez-Diaz L, Alejos O, Torres L and Tristan C 2007 Thermal effects on domain wall depinning from a single notch Phys. Rev. Lett. 99099901 (erratum)

[38] Martinez E, Lopez-Diaz L, Torres L, Tristan C and Alejos O 2007 Thermal effects in domain wall motion: micromagnetic simulations and analytical model Phys. Rev. B 75174409

[39] Duine R A and Morais Smith C 2008 Creep of current-driven domain-wall lines: effects of intrinsic versus extrinsic pinning Phys. Rev. B 77094434

[40] Martinez E, Lopez-Diaz L, Alejos O, Torres L and Carpentieri M 2009 Domain-wall dynamics driven by short pulses along thin ferromagnetic strips: micromagnetic simulations and analytical description Phys. Rev. B 79094430

[41] Lucassen M E, van Driel H J, Morais Smith C and Duine R A 2009 Current-driven and field-driven domain walls at nonzero temperature Phys. Rev. B 79224411

[42] Kim J-V and Burrowes C 2009 Influence of magnetic viscosity on domain wall dynamics under spin-polarized currents Phys. Rev. B 80214424

[43] Schieback C, Hinzke D, Kläui M, Nowak U and Nielaba P 2009 Temperature dependence of the current-induced domain wall motion from a modified Landau-Lifshitz-Bloch equation Phys. Rev. B $80214403-8$

[44] Le Maho Y, Kim J-V and Tatara G 2009 Spin-wave contributions to current-induced domain wall dynamics Phys. Rev. B 79174404

[45] Garcia-Sanchez F, Szambolics H, Mihai A P, Vila L, Marty A, Attané J-P, Toussaint J-Ch and Buda-Prejbeanu L D 2010 Effect of crystalline defects on domain wall motion under field and current in nanowires with perpendicular magnetization Phys. Rev. B 81134408

[46] Brown W F 1963 Thermal fluctuations of a single-domain particle Phys. Rev. 1301677

[47] Eltschka $M$ et al 2010 Nonadiabatic spin torque investigated using thermally activated magnetic domain wall dynamics Phys. Rev. Lett. 105056601

[48] Hänggi P, Talkner P and Borkovec M 1990 Reaction-rate theory: fifty years after Kramers Rev. Mod. Phys. 62 251-341

[49] Hubert A and Schäfer R 1998 Magnetic Domains: the Analysis of Magnetic Microstructures (Berlin: Springer)

[50] Kronmüller H and Parkin S S P (ed) 2008 Handbook of Magnetism and Advanced Magnetic Materials (Chichester: Wiley)

[51] Heyderman L J et al 2004 Fabrication of nanoscale magnetic ring structures and devices Microelectron Eng. 73-74 780-4

[52] Stöhr J, Wu Y, Hermsmeier B D, Samant M G, Harp G R, Koranda S, Dunham D and Tonner B P 1993 Element-specific magnetic microscopy with circularly polarized X-rays Science 259658

[53] Heyne L, Rhensius J, Bisig A, Krzyk S, Punke P, Klaüi M, Heyderman L J, Le Guyader L and Nolting F 2010 Direct observation of high velocity current induced domain wall motion Appl. Phys. Lett. 96032504

[54] Meier G, Bolte M, Eiselt R, Krüger B, Kim D H and Fischer P 2007 Direct imaging of stochastic domain-wall motion driven by nanosecond current pulses Phys. Rev. Lett. 98187202

[55] Bocklage L, Krüger B, Matsuyama T, Bolte M, Merkt U, Pfannkuche D and Meier G 2009 Dependence of magnetic domain-wall motion on a fast changing current Phys. Rev. Lett. 103197204

[56] Yamaguchi A, Ono T, Nasu S, Miyake K, Mibu K and Shinjo T 2004 Real-Space observation of current-driven domain wall motion in submicron magnetic wires Phys. Rev. Lett. 92077205

[57] Kläui M, Jubert P-O, Allenspach R, Bischof A, Bland J A C Faini G, Rüdiger U, Vaz C A F, Vila L and Vouille C 2005 Direct observation of domain-wall configurations transformed by spin currents Phys. Rev. Lett. 95026601

[58] Grollier J, Boulenc P, Cros V, Hamzić A, Vaurès A, Fert A and Faini G 2003 Switching a spin valve back and forth by current-induced domain wall motion Appl. Phys. Lett. $83509-11$

[59] Marrows C H 2005 Spin-polarised currents and magnetic domain walls Adv. Phys. 54 585-713

[60] Hayashi M, Thomas L, Rettner C, Moriya R and Parkin S S P 2007 Direct observation of the coherent precession of magnetic domain walls propagating along permalloy nanowires Nature Phys. 3 21-5

[61] Heyne L et al 2008 Relationship between nonadiabaticity and damping in permalloy studied by current induced spin structure transformations Phys. Rev. Lett. 100066603

[62] Hayashi M, Thomas L, Rettner C, Moriya R, Bazaliy Y B and Parkin S S P 2007 Current driven domain wall velocities exceeding the spin angular momentum transfer rate in permalloy nanowires Phys. Rev. Lett. 98037204

[63] Hayashi M, Thomas L, Bazaliy Ya B, Rettner C, Moriya R, Jiang X and Parkin S S P 2006 Influence of current on field-driven domain wall motion in permalloy nanowires from time resolved measurements of anisotropic magnetoresistance Phys. Rev. Lett. 96197207

[64] Parkin S S P, Hayashi M and Thomas L 2008 Magnetic domain-wall racetrack memory Science 320 190-4

[65] Togawa Y, Kimura T, Harada K, Matsuda T, Tonomura A, Otani Y and Akashi T 2008 Current-excited magnetization reversal under in-plane magnetic field in a nanoscaled ferromagnetic wire Appl. Phys. Lett. 92012505

[66] Ohno $\mathrm{H}$ and Dietl T 2008 Spin-transfer physics and the model of ferromagnetism in (Ga,Mn)As J. Magn. Magn. Mater 320 1293-9

[67] Matsukura F, Chiba D and Ohno H 2008 Spintronics vol 82 (Amsterdam: Elsevier) pp 207-40

[68] Chiba D, Yamanouchi M, Matsukura F and Ohno H 2009 Current-induced domain wall motion in ferromagnetic semiconductor structures Magn. Japan 4390

[69] Sobolev V L 1998 Internal structure of a domain wall in ultrathin magnetic film J. Magn. Magn. Mater. 177-181 195-6

[70] Mougin A, Cormier M, Adam J P, Metaxas P J and Ferre J 2007 Domain wall mobility, stability and walker breakdown in magnetic nanowires Europhys. Lett. 7857007

[71] Fukami S, Suzuki T, Ohshima N, Nagahara K and Ishiwata N 2008 Intrinsic threshold current density of domain wall motion in nanostrips with perpendicular magnetic anisotropy for use in low-write-current mrams IEEE Trans. Magn. 44 2539-42

[72] Suzuki T, Fukami S, Ohshima N, Nagahara K and Ishiwata N 2008 Analysis of current-driven domain wall motion from pinning sites in nanostrips with perpendicular magnetic anisotropy J. Appl. Phys. 103113913

[73] Fukami S, Suzuki T, Ohshima N, Nagahara K and Ishiwata N 2008 Micromagnetic analysis of current driven domain wall motion in nanostrips with perpendicular magnetic anisotropy J. Appl. Phys. 103 07E718 
[74] Fukami S, Nakatani Y, Suzuki T, Nagahara K, Ohshima N and Ishiwata N 2009 Relation between critical current of domain wall motion and wire dimension in perpendicularly magnetized Co/Ni nanowires Appl. Phys. Lett. 95232504

[75] Jung S W, Kim W, Lee T D, Lee K J and Lee H W 2008 Current-induced domain wall motion in a nanowire with perpendicular magnetic anisotropy Appl. Phys. Lett. 92 202508-3

[76] Martinez E, Lopez-Diaz L, Alejos O and Torres L 2009 Thermally activated domain wall depinning in thin strips with high perpendicular magnetocrystalline anisotropy, domain wall thermal effect J. Appl. Phys. 106043914

[77] Garcia-Sanchez F, Szambolics H, Mihai A P, Vila L, Marty A, Attané J-P, Toussaint J-Ch and Buda-Prejbeanu L D 2010 Effect of crystalline defects on domain wall motion under field and current in nanowires with perpendicular magnetization, domain wall thermal effect Phys. Rev. B 81134408

[78] Yan M, Kokay A, Gliga S and Hertel R 2010 Beating the walker limit with massless domain walls in cylindrical nanowires Phys. Rev. Lett. 104057201

[79] Heyne L et al 2009 Geometry-dependent scaling of critical current densities for current-induced domain wall motion and transformations Phys. Rev. B 80184405

[80] Malinowski G et al 2010 Current-induced domain wall motion in ni $80 \mathrm{Fe} 20$ nanowires with low depinning fields J. Phys. D: Appl. Phys. 43045003

[81] Mizukami S, Sajitha E P, Watanabe D, Wu F, Miyazaki T, Naganuma H, Oogane $\mathrm{M}$ and Ando Y 2010 Gilbert damping in perpendicularly magnetized $\mathrm{Pt} / \mathrm{Co} / \mathrm{Pt}$ films investigated by all-optical pump-probe technique Appl. Phys. Lett. 96152502

[82] Bisig A et al 2010 Direct imaging of current induced magnetic vortex gyration in an asymmetric potential well Appl. Phys. Lett. 96152506

[83] Yamaguchi A, Nasu S, Tanigawa H, Ono T, Miyake K, Mibu K and Shinjo T 2005 Effect of joule heating in current-driven domain wall motion Appl. Phys. Lett. 86012511

[84] You C-Y, Sung I M and Joe B-K 2006 Analytic expression for the temperature of the current-heated nanowire for the current-induced domain wall motion Appl. Phys. Lett. 89222513

[85] You C Y and Ha S S 2007 Temperature increment in a current-heated nanowire for current-induced domain wall motion with finite thickness insulator layer Appl. Phys. Lett. 91022507

[86] Laufenberg M, Bührer W, Bedau D, Melchy P-E, Kläui M, Vila L, Faini G, Vaz C A F, Bland J A C and Rüdiger U 2006 Temperature dependence of the spin torque effect in current-induced domain wall motion Phys. Rev. Lett. 97046602

[87] Boulle O, Kimling J, Warnicke P, Kläui M, Rüdiger U, Malinowski G, Swagten H J M, Koopmans B, Ulysse C and Faini G 2008 Nonadiabatic spin transfer torque in high anisotropy magnetic nanowires with narrow domain walls Phys. Rev. Lett. 101216601

[88] Cormier M, Mougin A, Ferré J, Thiaville A, Charpentier N, Piéchon F, Weil R, Baltz V and Rodmacq B 2010 Effect of electrical current pulses on domain walls in $\mathrm{Pt} / \mathrm{Co} / \mathrm{Pt}$ nanotracks with out-of-plane anisotropy: spin transfer torque versus joule heating Phys. Rev. B 81024407

[89] Hayashi M, Thomas L, Rettner C, Jiang X and Parkin S S P 2007 Temporal evolution of exchange bias in spin-valve nanowires on the nanosecond time scale Europhys. Lett. 7867006

[90] Thomas L, Hayashi M, Jiang X, Rettner R C and Parkin S S P 2008 Perturbation of spin-valve nanowire reference layers during domain wall motion induced by nanosecond-long current pulses Appl. Phys. Lett. 92112504
[91] Burrowes $C$ et al 2010 Non-adiabatic spin-torques in narrow magnetic domain walls Nature Phys. 6 17-21

[92] Hankemeier S, Sachse K, Stark Y, Frömter R and Oepen H P 2008 Ultrahigh current densities in permalloy nanowires on diamond Appl. Phys. Lett. 92242503

[93] Boulle O et al 2009 J. Appl. Phys. $10507 \mathrm{C} 106$

[94] Yamanouchi M, Chiba D, Matsukura F, Dietl T and Ohno H 2006 Velocity of domain-wall motion induced by electrical current in the ferromagnetic semiconductor $(\mathrm{Ga}, \mathrm{Mn}) \mathrm{As}$ Phys. Rev. Lett. 96096601

[95] Heinen J, Boulle O, Rousseau K, Malinowski G, Klaüi M, Swagten H J M, Koopmans B, Ulysse C and Faini G 2010 Current-induced domain wall motion in $\mathrm{Co} / \mathrm{Pt}$ nanowires: separating spin torque and oersted-field effects Appl. Phys. Lett. 96202510

[96] Ravelosona D, Lacour D, Katine J A, Terris B D and Chappert C 2005 Nanometer scale observation of high efficiency thermally assisted current-driven domain wall depinning Phys. Rev. Lett. 95117203

[97] Feigenson M, Reiner J W and Klein L 2008 Current-induced magnetic instability in srruo3 J. Appl. Phys. $10307 \mathrm{e} 741$

[98] Aziz A, Bending S J, Roberts H G, Crampin S, Heard P J and Marrows C H 2006 Angular dependence of domain wall resistivity in artificial magnetic domain structures Phys. Rev. Lett. 97206602

[99] Ravelosona D, Mangin S, Katine J A, Fullerton E E and Terris B D 2007 Threshold currents to move domain walls in films with perpendicular anisotropy Appl. Phys. Lett. 90072508

[100] Songtian Li, Nakamura H, Kanazawa T, Liu X and Morisako A 2010 Current-Induced domain wall motion in $\mathrm{TbFeCo}$ wires with perpendicular magnetic anisotropy IEEE Trans. Magn. 46 1695-8

[101] Suzuki T, Fukami S, Nagahara K, Ohshima N and Ishiwata N 2009 Evaluation of scalability for current-driven domain wall motion in a Co/Ni multilayer strip for memory applications IEEE Trans. Magn. 45 3776-9

[102] Tanigawa $\mathrm{H}$ et al 2009 Domain wall motion induced by electric current in a perpendicularly magnetized $\mathrm{Co} / \mathrm{Ni}$ Nano-Wire Appl. Phys. Express 2053002

[103] Koyama T et al 2011 Observation of the intrinsic pinning of a magnetic domain wall in a ferromagnetic nanowire Nature Mater. 10 194-7

[104] Lee J-C, Kim K-J, J R, Moon K-W, Yun S-J, Gim G-H, Lee K-S, Shin K-H, Lee H-W and Choe S-B 2009 Roles of adiabatic and nonadiabatic spin transfer torques on magnetic domain wall motion arXiv: 1006.1216v1

[105] San Emeterio Alvarez L, Wang K-Y, Lepadatu S, Landi S, Bending S J and Marrows C H 2010 Spin-transfer-torque-assisted domain-wall creep in a co/pt multilayer wire Phys. Rev. Lett. 104137205

[106] Miron I M, Zermatten P-J, Gaudin G, Auffret S, Rodmacq B and Schuhl A 2009 Domain wall spin torquemeter Phys. Rev. Lett. 102137202

[107] Feigenson M, Reiner J W and Klein L 2007 Efficient current-induced domain-wall displacement in srruo3 Phys. Rev. Lett. 98247204

[108] Nguyen A K, Skadsem H J and Brataas A 2007 Giant current-driven domain wall mobility in $(\mathrm{Ga}, \mathrm{Mn})$ As Phys. Rev. Lett. 98146602

[109] Garate I, Gilmore K, Stiles M D and MacDonald A H 2009 Nonadiabatic spin-transfer torque in real materials Phys. Rev. B 79104416

[110] Hals K M D, Nguyen A K and Brataas A 2009 Intrinsic coupling between current and domain wall motion in (Ga,Mn)As Phys. Rev. Lett. 102256601

[111] Miron I M, Gaudin G, Auffret S, Rodmacq B, Schuhl A Pizzini S, Vogel J and Gambardella P 2010 Current-driven 
spin torque induced by the rashba effect in a ferromagnetic metal layer Nature Mater. $9230-4$

[112] Ban Y and Tatara G 2009 Spin-transfer torque in disordered weak ferromagnets Phys. Rev. B 80184406

[113] Tatara G, Kohno H and Shibata J 2008 Theory of domain wall dynamics under current J. Phys. Soc. Japan 77031003
[114] Adam J-P, Vernier N, Ferré J, Thiaville A, Jeudy V, Lemaître A, Thevenard L and Faini G 2009 Nonadiabatic spin-transfer torque in (Ga,Mn)As with perpendicular anisotropy Phys. Rev. B 80193204

[115] Foros J, Brataas A, Tserkovnyak Y and Bauer G E W 2008 Current-induced noise and damping in nonuniform ferromagnets Phys. Rev. B 78140402 